\title{
The role of modified biophysical profile in predicting perinatal outcome in high risk pregnancies
}

\author{
Jankidevi S. Borade*, Sushma P. Sharma
}

Department of Obstetrics and Gynecology, MIMER Medical College, Talegaon, Pune, Maharashtra, India

Received: 21 March 2018

Accepted: 21 April 2018

\section{*Correspondence:}

Dr. Jankidevi S. Borade,

E-mail: jankimane@gmail.com

Copyright: $\odot$ the author(s), publisher and licensee Medip Academy. This is an open-access article distributed under the terms of the Creative Commons Attribution Non-Commercial License, which permits unrestricted non-commercial use, distribution, and reproduction in any medium, provided the original work is properly cited.

\section{ABSTRACT}

Background: The goal of antepartum fetal surveillance is early identification of the compromised fetus and timely intervention when the fetus is at risk, but still in an uncompromised state. Modified Biophysical Profile (MBPP) includes Amniotic Fluid Index (AFI) and non stress test (NST). AFI is a marker of long term placental function and NST is a marker of short term fetal condition. The aim of this study is to assess the role of Modified Biophysical Profile in high risk pregnancies and assess perinatal outcome and to study the impact of NST and AFI individually in high risk pregnancies.

Methods: 100 ANC patients with high risk factors were evaluated with modified biophysical profile 37 week onwards with non stress test (NST) for 20 mins and amniotic fluid index (AFI) with 4 quadrant technique. High risk pregnancies include preeclampsia, IUGR, oligohydramnios, postdated pregnancy, etc. and various parameters were assessed to determine perinatal and maternal morbidity. All parameters were statistically analyzed.

Results: The above study states that need for LSCS, intrapartum fetal distress, meconium stained liquor, APGAR score, need for neonatal resuscitation and perinatal morbidity were definitely higher in cases with abnormal MBPP.

Conclusions: Thus MBPP is an easy, cost effective and time saving measure and hence can be used as a primary antepartum fetal surveillance test to predict perinatal outcome and provide timely intervention in high risk pregnancies.

Keywords: Antepartum fetal surveillance, AFI, MBPP, NST

\section{INTRODUCTION}

It is a known fact that no health problem is more significance to a nation than maternal health and perinatal morbidity. The average perinatal mortality in India is 16 per 1000 live births. ${ }^{1}$

Many maternal high risk conditions like pre-eclampsia, oligohyramnios, anemia lead to adverse neonatal outcome. Timely intervention in these pregnancies can bring down the perinatal morbidity and mortality.

Fetal biophysical profile is a well established method of antepartum surveillance in high risk pregnancies.
Classical biophysical profile was described by Manning et al. $^{2}$ It includes 5 parameters- fetal tone, breathing movements, gross body movements, amniotic fluid volume, and non stress test. Hence it's more time consuming, cumbersome and expensive.

Modified biophysical profile (MBPP) was first described by Nageotte et al. ${ }^{3,4}$ It includes Amniotic Fluid Index (AFI) and non stress test (NST).

AFI is a marker of long term placental function and NST is a marker of short term fetal condition. MBPP is hence less time consuming and easier to perform. 
Hence in this study modified biophysical profile is used as a primary marker for fetal surveillance in high risk pregnancies to study its effectiveness in perinatal outcome.

Objective of present study was to evaluate the role of modified fetal biophysical profile in high risk pregnancies for predicting the perinatal outcome.

\section{METHODS}

This was a prospective observational study including 100 high risk pregnancies. Pregnant women with high risk factors in ANC OPD admitted in ward or in emergency in Dept. Obstetrics and Gynaecology in Dr. BSTRH Hospital, Talegaon who fulfilled the inclusion and exclusion criteria were enrolled for the study.

Written, informed, valid consent was taken in the language they best understood. Detailed history of the pregnant women was taken and thorough clinical examination including vital parameters, systemic examination, and obstetric examination was done and all routine investigations were done. The patients with high risk factors were evaluated with modified biophysical profile 37 week onwards with Non stress test (NST) FOR 20 mins and Amniotic Fluid Index (AFI) with 4 quadrant technique. ${ }^{5}$

NST was performed in supine position and fetal heart sound, fetal movements, uterine contractions, if any were recorded. NST was considered reactive if more than two fetal movements with accelerations of more than 15 beats/ minutes lasting for more than 15 seconds with good beat to beat variability with no decelerations are seen in 20 mins. If NST was not reactive, the fetus was stimulated with VAST (Vibro Acoustic Stimulator) and the NST was repeated. If the result is still not reactive for 40mins, it was labelled a non reactive NST. Real time ultrasound was done and AFI was calculated by the four quadrant technique.

As described by Phelan et al. ${ }^{6}$ AFI will be considered abnormal if less than or equal to 5 and more than or equal to 25. Patients were followed up till delivery and perinatal outcome was assessed and attributed to the last MBPP before delivery.

\section{Reactive NST}

- $\quad$ Baseline 110-150 bpm

- Amplitude baseline variability of 5-25bpm

- Two or more accelerations of $15 \mathrm{bpm}$ lasting for $15 \mathrm{secs}$

- Absence of decelerations. $^{7}$

\section{Non Reactive NST}

- Baseline less than 100 or above 170

- Baseline variability of less than $5 \mathrm{bpm}$
- $\quad$ Sinusoidal pattern

- Repeated, prolonged and severe variable decelerations

- Periodically occurring repeated late decelerations.7

Perinatal outcome in cases of

- Normal AFI and NST

- Normal AFI but non reactive NST

- Reactive NST but abnormal AFI

- Abnormal AFI and Non reactive NST

\section{Inclusion criteria}

Singleton pregnancies with cephalic pregnancies with gestational age of $37 \mathrm{wks}$ or more with either of the below:

- Pre eclampsia

- Eclampsia

- Anemia

- Oligohydramnios

- Pregnancy after 40 wks

- Decreased fetal movements

- Previous still birth

- Clinically suspected IUGR

- Diabetes mellitus, Gestational diabetes

\section{Exclusion criteria}

- $\quad$ Preterm fetus

- Fetuses with congenital anomalies

- Intrauterine deaths

- Multifetal pregnancies

\section{Parameters to be assessed}

- Mode of delivery

- Nature of liquor

- Fetal distress

- Apgar at birth

- Need for neonatal resuscitation

- Perinatal morbidity

For study purpose, Patients were divided into 4 groups NST and AFI both were normal in 67 cases (Group A). NST was normal and AFI was abnormal in 22 patients (Group B). AFI was normal and NST was abnormal in 5 patients (Group C). Both parameters were abnormal in 6 patients (Group D).

\section{Statistical analysis}

A descriptive statistics i.e. frequencies and percentages will be calculated. Chi square test will be used to test the associations between the variables. Binary logistic regression will be applied to measure the risk associated 
with modified biophysical profile. $\mathrm{Z}$ test (proportion test) will be used to find significant differences.

\section{RESULTS}

The patients were categorized according to the gestational age in weeks; it was found that $20 \%$ of the cases were between $37-38$ weeks. $32 \%$ of the cases were between the gestational ages of 39-40 weeks. Majority of the cases were above 40 weeks of gestation. Those who were above 40 weeks, all were prolonged, none were post term.

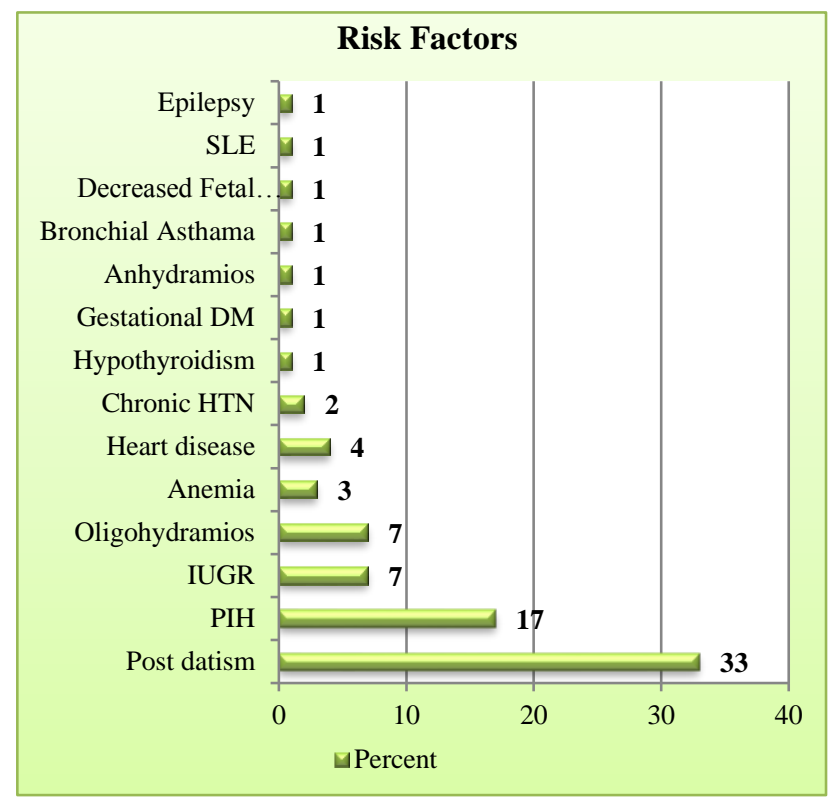

*Some patients had more than one risk factor, hence the total is 119 and not 100.

Figure 1: High risk factors.

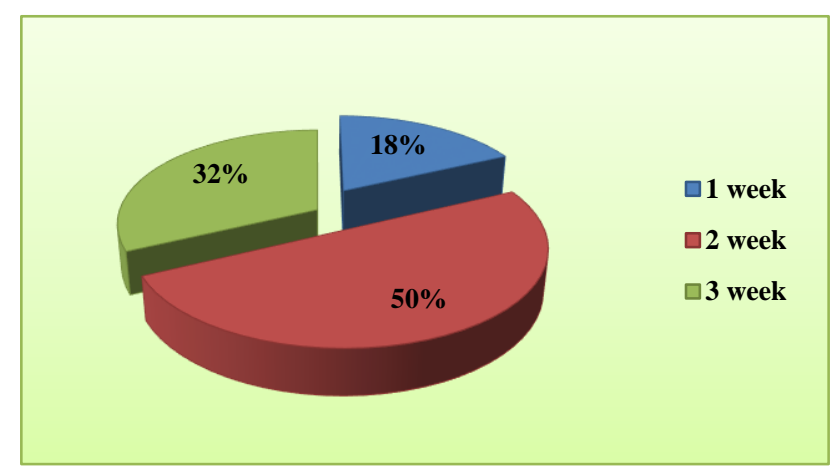

Figure 2: Weekly follow-up of MBPP.

Figure 1 shows the risk factors with which the patients presented. Hypertensive disorders in pregnancy which included 30 patients with pre-eclampsia and 3 with chronic hypertension. The majority of patients (40) were prolonged pregnancy (with no other risk factor). 16 patients had IUGR while 12 had Oligohyramnios and one with anhydramnios as their high risk. There were 6 patients with anemia and 4 with heart disease. 2 patients were with hypothyroidism while 2 were with gestational diabetes mellitus. There were 1 patient each with bronchial asthma, decreased fetal movements, SLE and epilepsy.

Figure 2 shows that out of the 22 follow up cases, majority of them, 11 cases $(50 \%)$ had followed up weekly for two weeks, while 7 (31.8\%) had weekly follow up for 3 weeks. 4 cases $(18.2 \%)$ had a MBPP one week before delivery.

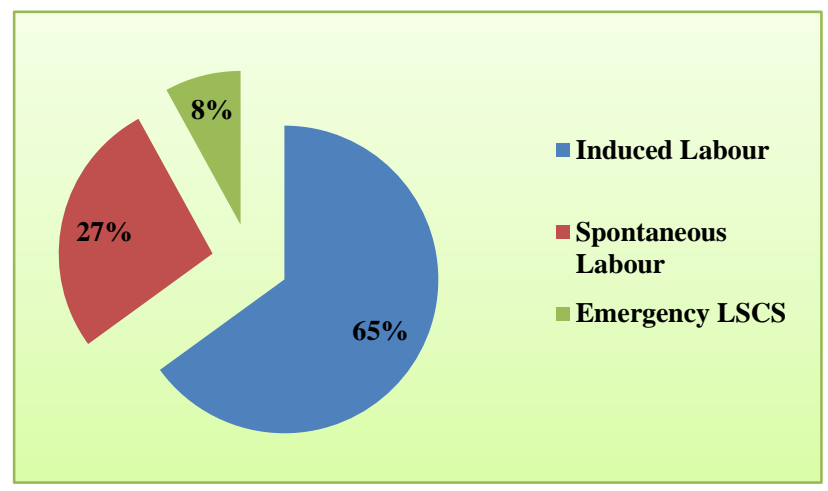

Figure 3: Type of labour.

Figure 3 Out of the 92 patients who were given trial of labour, 65 cases $(70.7 \%)$ were induced while 27 patients $(29.3 \%)$ had spontaneous onset of labour. 8 patients were taken up for emergency LSCS directly due to non reactive NST.

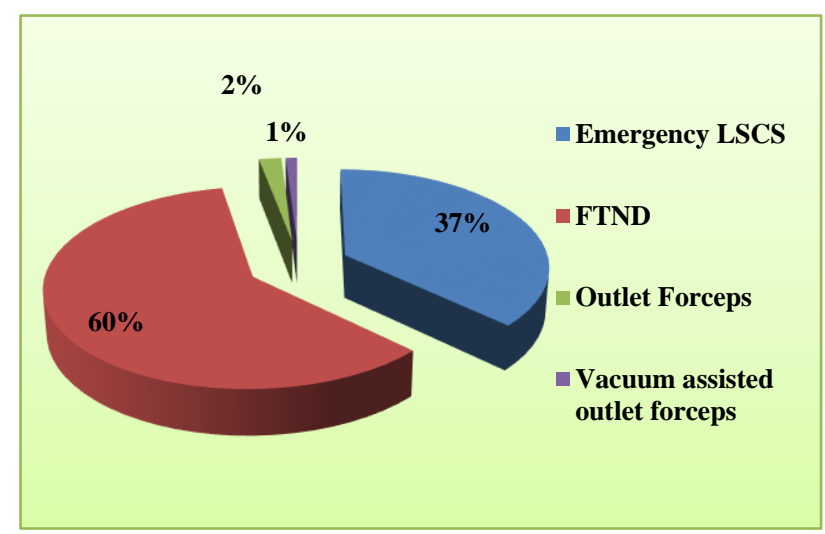

*Total is 38 as 1 patient had intrapartum fetal distress and also meconium stained liquor.

Figure 4: Mode of delivery.

Figure 4 says of 100 patients 63 of them had vaginal delivery and 37 of them had emergency caesarean section. Out of the 63 patients who had vaginal delivery 60 of them had full term normal delivery, 2 of them had Outlet Forceps, while 1 had vacuum followed by outlet forceps.

Figure 5 shows that of the 37 cases who underwent caesarean section, 29 were given trial of labour while 8 were taken up for emergency LSCS for non reactive NST 
with/without oligohydraminos and without trial of labour. 25 of them $(66.5 \%)$ had intrapartum fetal distress while 8 patients $(20.5 \%)$ had their indication as non reactive NST. 3 patients $(8.1 \%)$ had meconium stained amniotic fluid as their indication. One patient $(2.7 \%)$ had non progress of labour while one $(2.7 \%)$ had failure of induction as their indication. Total is 38 as 1 patient had intrapartum fetal distress and also meconium stained liquor.

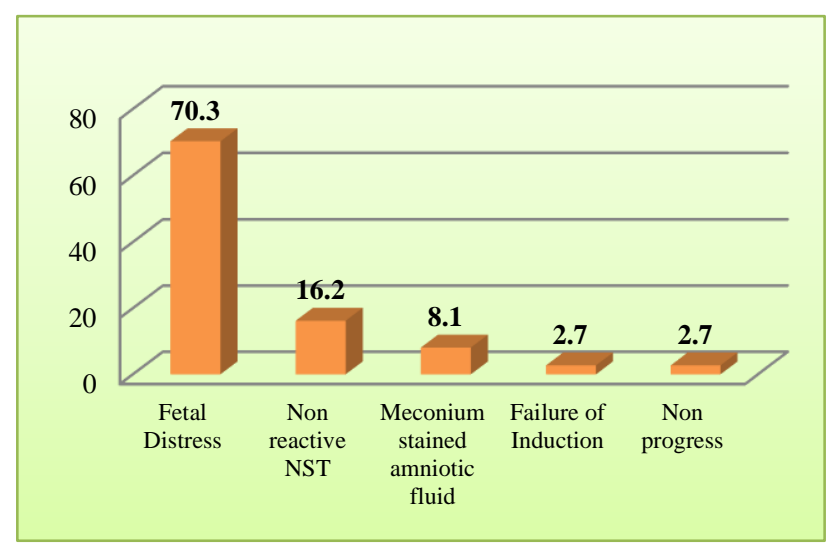

Figure 5: Indication for LSCS.

\section{MBPP and mode of delivery}

Amongst 67 patients with normal MBPP, 49 (74.2\%) patients had vaginal delivery while 17 (25.5\%) patients had LSCS. While, out of 33 patients with abnormal MBPP, 13 (41\%) had vaginal delivery but 20 (59\%) patients needed LSCS, $\mathrm{p}<0.01$.

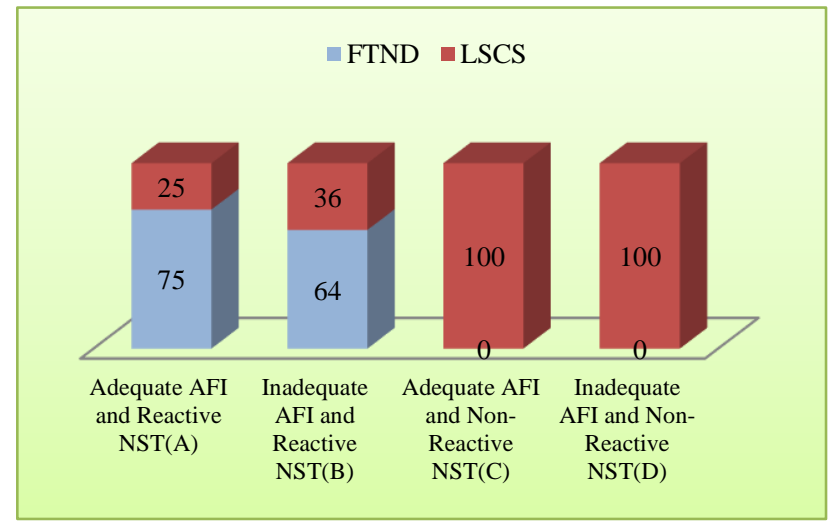

Figure 6: Individual parameters of MBPP and its effect on mode of delivery.

Among the modified biophysical profiles done in 100 patients, when both parameters (NST and AFI) were normal (67 patients) $17(25.5 \%)$ patients underwent LSCS and $49(74.2 \%)$ patients had vaginal delivery (Figure 6). When both parameters were abnormal (6 patients) all 6 $(100 \%)$ patients underwent LSCS. When NST was normal and only AFI was abnormal (22patients), $8(36.3 \%)$ patients had vaginal delivery \& $14.3(63.6 \%)$ of them underwent LSCS. When AFI was normal and NST was abnormal (5 patients) all 5 patients (100\%) underwent LSCS $(\mathrm{P}<0.01)$.

\section{MBPP and presence of meconium stained liquor}

When the MBPP was normal, out of 67 patients 11(17\%) patients had thick meconium stained liquor; while when MBPP is abnormal 9 out of 34 (26\%) patient had meconium stained liquor ( $\mathrm{P}$ value -0.29 ).

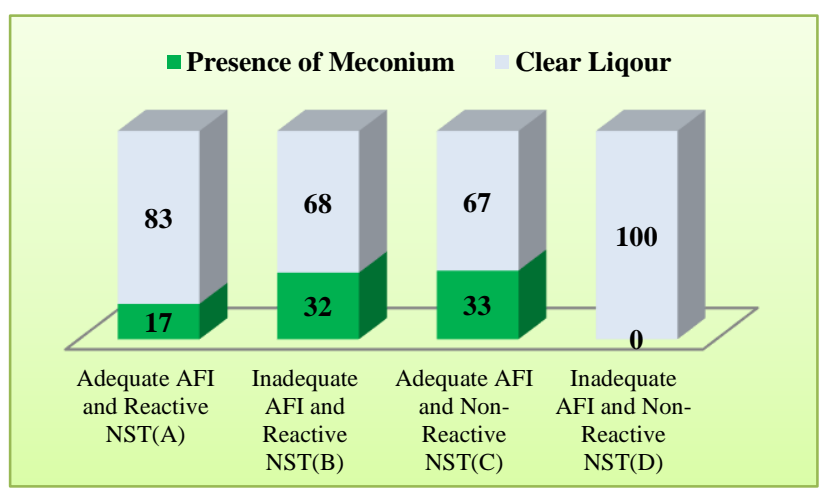

Figure 7: Last MBPP and its role in predicting Meconium stained liquor.

Figure 7 out of 100 patients' meconium staining of liquor was observed among 20 cases (12 thick and 8 thin). When both parameters (NST and AFI) were normal out of 67 patients, $11(17 \%)$ patients had thick meconium stained liquor, when both parameters were abnormal out of 6 , no patient had meconium stained liquor. When NST was normal and AFI was abnormal, 7 (32\%) patients of 22 had meconium stained liquor and when AFI was normal and NST was abnormal $2(32 \%)$ patients had thick meconium stained liquor of 5 patients ( $\mathrm{P}$ value 0.21 ). Out of 100 patients, 92 patients had trial of labour, while 8 directly went in for LSCS. Intrapartum fetal distress was observed among 31 cases. 25 patients had LSCS while 6 delivered vaginaly. 26 patients had late decelerations, 3 had variable decelerations while 2 had persistent fetal tachycardia.

\section{MBPP and intrapartum fetal distress}

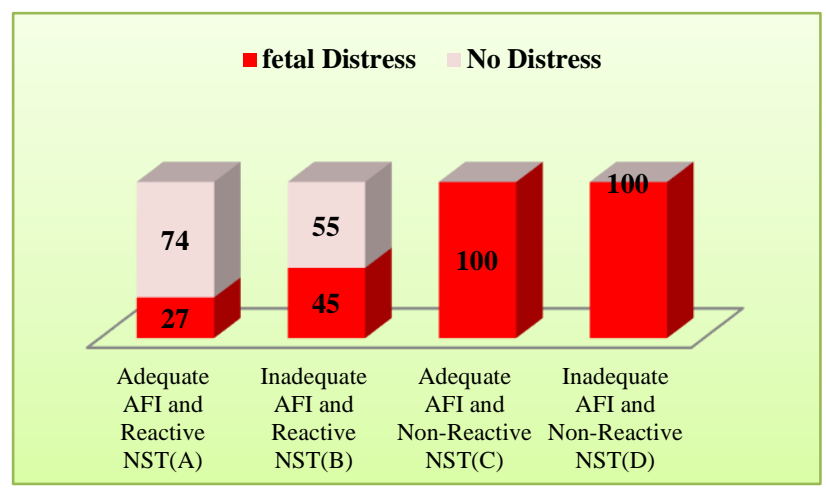

Figure 8: Individual components of MBPP and presence of intrapartum fetal distress. 
When MBPP was normal, 18(27\%) out of 67 patients had intrapartum fetal distress, while in those it was abnormal, 13 out of $25(52 \%)$ had fetal distress $(\mathrm{P}<0.01)$.

Figure 8 shows when both parameters (NST and AFI) were normal out of 67 patients, $18(27 \%)$ patients had intrapartum fetal distress, when both parameters were abnormal out of 1 patient was given trial of labour and she developed intrapartum fetal distress (100\%). When NST was normal and AFI was abnormal, 10 (45\%) patients of 22 had intrapartum fetal distress. When AFI was normal and NST was abnormal 2 patients were given trial of labour and both $(100 \%)$ patients had intrapartum fetal distress $(\mathrm{P}<0.01)$.

\section{MBPP and APGAR score}

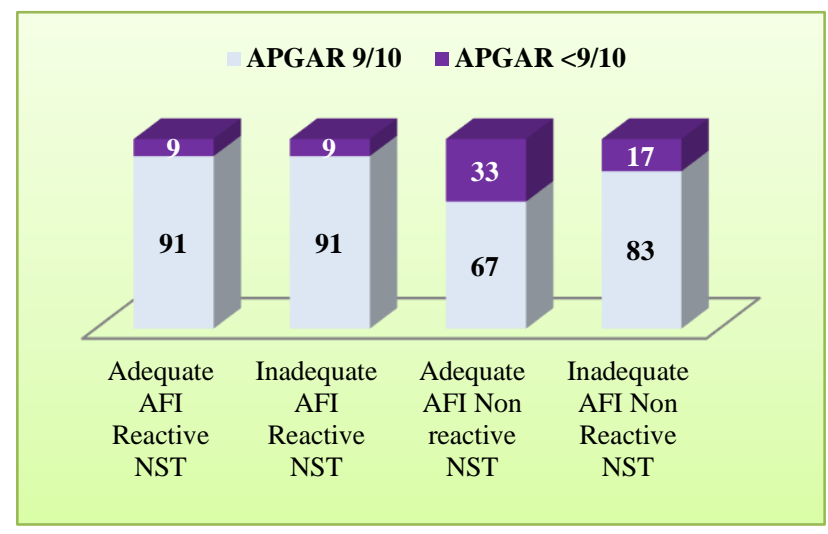

Figure 9: Individual components of MBPP and APGAR score.

Those with a normal MBPP 6 out of 67 patients had APGAR $<9(9 \%)$, While 5 out of 33 with those of abnormal MBPP had APGAR <9 $(14.2 \%)(\mathrm{P}<0.69)$.

Among the 100 cases included in the study, APGAR score of $<9$ was observed among 11 cases (Figure 9). When both parameters (NST and AFI) were normal 6 (9\%) patient had APGAR score of $<9$, when both parameters were abnormal 1(16\%) patients had APGAR score of $<9$ out of 6 , when NST was normal and AFI was abnormal 2(9\%) of the 22 patients had APGAR score of $<9$ and when AFI was normal and NST was abnormal $2(33 \%)$ patients had APGAR score of $<9$ out of 5 (P $0.32)$

\section{Neonatal outcome}

Out of the 11 babies with APGAR <9/10, 8 babies had APGAR 8/10. Only 1 NICU admission in view of meconium aspiration syndrome was done, who was discharged on day 3. All other babies cried on resuscitation and were with their mothers.

3 babies had APGAR <8/10. They needed NICU admission for 4-5 days but were discharged without any neurological deficit.

\section{MBPP and need for neonatal resuscitation}

Resuscitation was needed for 40 out of the 100 babies born. Stimulation followed by suctioning was needed in $19(47.5 \%)$ while $21(52.5 \%)$ babies needed intratracheal suctioning.

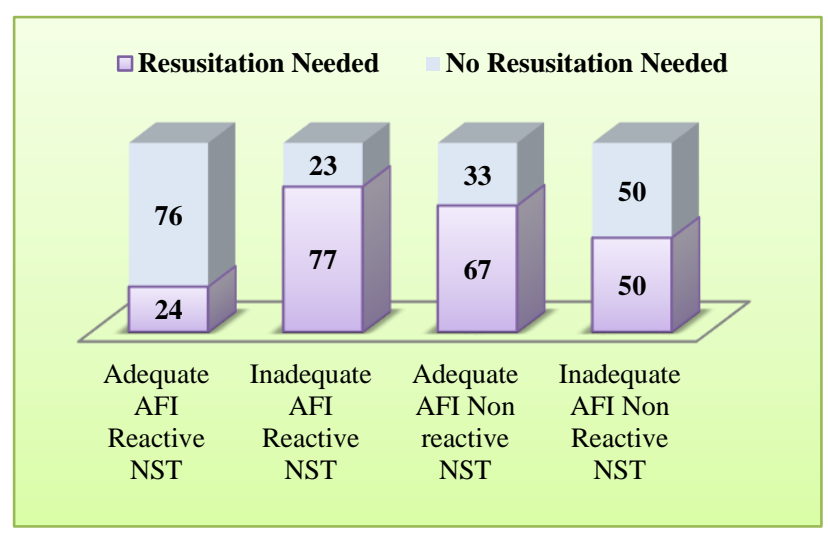

Figure 10: Individual component of last MBPP and need for neonatal resuscitation.

When the MBPP is normal, 17 (24.2\%) out of 67 babies needed resuscitation while $24(70.5 \%)$ out of 34 babies with abnormal MBPP needed resuscitation $\mathrm{P}<0.01$.

Figure 10 explains that among the 100 cases included in the study, neonatal resuscitation was needed by 40 babies. When both parameters (NST and AFI) were normal $17(24 \%)$ babies needed resuscitation, when both parameters were abnormal $3(50 \%)$ of 6 babies needed resuscitation, when NST was normal and AFI was abnormal, $17(77 \%)$ of the 22 babies needed resuscitation and when AFI was normal and NST was abnormal 3 $(67 \%)$ of 5 babies needed resuscitation $(\mathrm{p}<0.01)$.

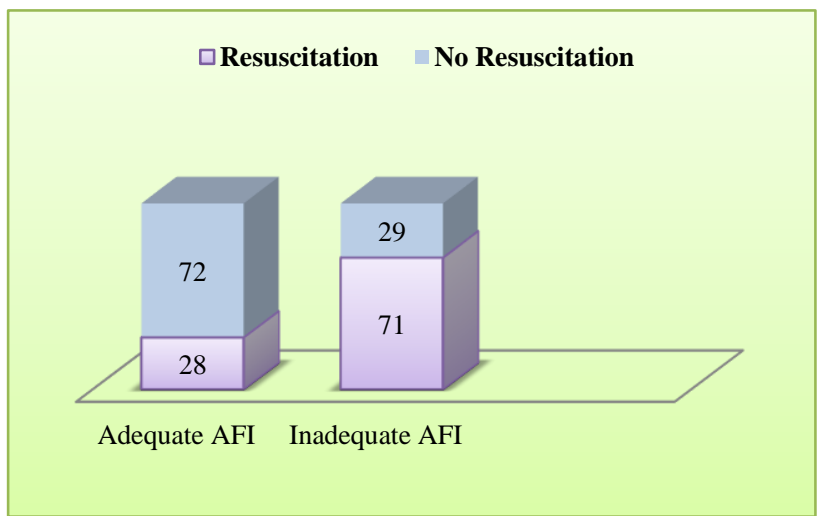

Figure 11: Last AFI and need of neonatal resuscitation.

Figure 11 shows $20(27.2 \%)$ out of 72 babies with an adequate AFI needed resuscitation; while 20 (71.4\%) out of 28 babies with inadequate AFI needed resuscitation after birth $(\mathrm{p}<0.01)$. 


\section{Neonatal outcome}

4 babies with meconium stained amniotic fluid needed NICU admission after intratracheal suctioning and had respiratory distress. Babies were given antibiotics and were discharged in 4-5 days. No neurological deficit. 2 babies with APGAR <6/10 needed IPPR for $3-5$ minutes and were kept in NICU on CPAP. Both were discharged on day 5. No baby had any neurological deficit on discharge.

\section{MBPP and perinatal morbidity}

Out of the 100 babies born, 40 had perinatal morbidity. 8 babies had low birth weight, 10 had meconium aspiration syndrome and 22 had respiratory distress.

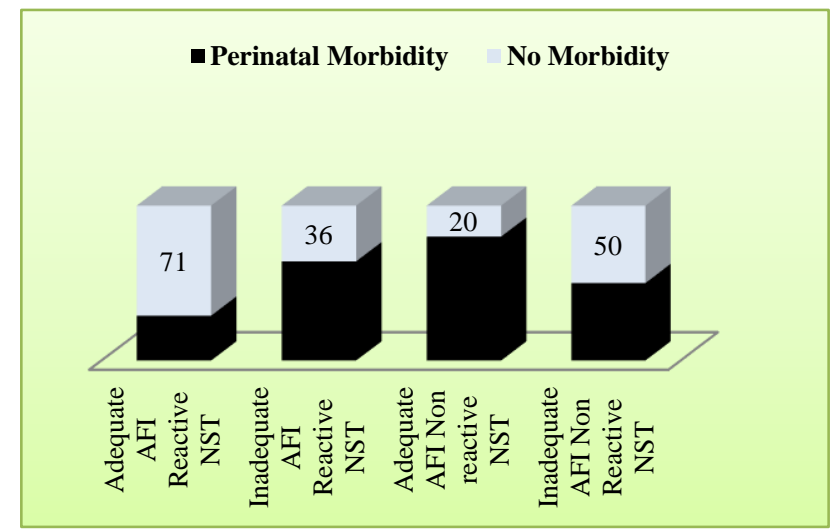

Figure 12: Individual component of MBPP and perinatal morbidity.

Out of the 67 babies with normal MBPP, 19 (28.7\%) babies had perinatal morbidity while $21(61.7 \%)$ out of 33 babies with abnormal MBPP had some perinatal morbidity $(\mathrm{P}<0.01)$.

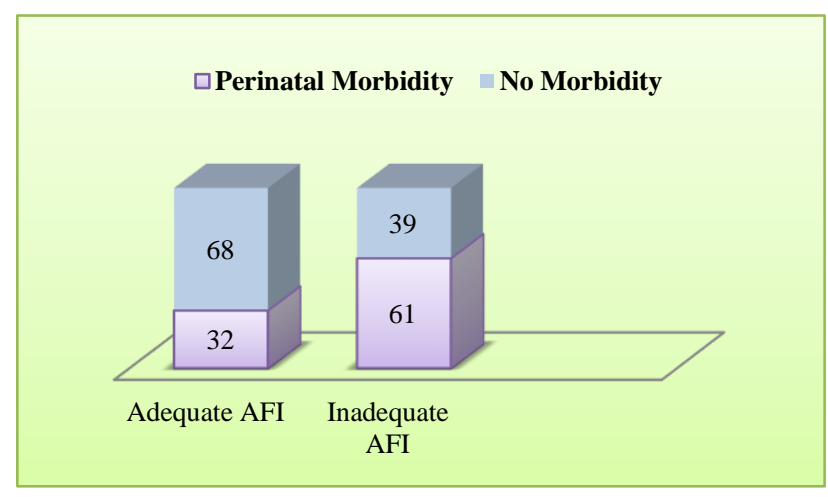

Figure 13: AFI and perinatal morbidity.

Figure 12 shows out of the 67 patients with adequate AFI and reactive NST, $19(29 \%)$ babies had perinatal morbidity.14 $(63.6 \%)$ out of 22 patients with inadequate AFI and non reactive NST had perinatal morbidity. 4 (80\%) out of 5 patients with non reactive NST but normal AFI had some type of perinatal morbidity. While $3(50 \%)$ out of 6 patients with non reactive NST with abnormal AFI had perinatal morbidity (P -0.014).

Figure 13 shows out of the all the 100 babies, 17 (61\%) of those with inadequate AFI has perinatal morbidity, while $23(32 \%)$ with adequate AFI had the same $(\mathrm{P}<0.01)$.

\section{DISCUSSION}

The risks of still births in prolonged pregnancy have been weighed against the complications of iatrogenic prematurity by induction of labour by Edmunds et al. ${ }^{8}$ As majority of the cases were of prolonged pregnancy, MBPP can be used to asses fetal well being and hence it can be used for timed induction of labour. Figure 1 as the fetal well being is assured by MBPP, the gestational age of delivery can be optimised to prevent iatrogenic prematurity in such high risk conditions. Figure 2 shows that MBPP can be used for follow up of high risk cases to ascertain fetal well being. Figure 3 show that the number of patients in whom labour was induced were statistically significant. Thus MBPP is rightly used for timed induction.

Hence, can be used as a primary antepartum fetal surveillance test to predict perinatal outcome and provide timely intervention in high risk pregnancies as also stated by Donald et al, Matsura et al and Arias et al. ${ }^{9-11}$

Figure 4 the rate of LSCS is higher than usual, but may be attributed to maternal high risk factors. Figure 5 shows that most common indication for LSCS in this study is Fetal distress either intrapartum or antepartum. Perinatal outcome is unfavourable in cases with non reactive NST as stated by Raouf S et al. ${ }^{12}$ Hence, continuous electronic fetal monitoring should be done in anticipation of fetal distress for cases with abnormal MBPP.

Table 1: Comparison of thick meconium staining of liquor with other study groups.

\begin{tabular}{|c|c|c|}
\hline Studies & $\begin{array}{l}\text { No. of patients } \\
(\%)\end{array}$ & P value \\
\hline Eden et al (337) ${ }^{13}$ & $52(15.4)$ & $<0.05 \mathrm{~S}$ \\
\hline Patil et al $(650)^{14}$ & $71(11.5)$ & $<0.05 \mathrm{~S}$ \\
\hline Present study (100) & $9(26 \%)$ & $\begin{array}{l}<0.05 \\
\text { Significant }\end{array}$ \\
\hline
\end{tabular}

Table 2: Comparison of incidence of LSCS for fetal distress with other study groups.

\begin{tabular}{|lll|}
\hline Studies & $\begin{array}{l}\text { No. of patients } \\
(\%)\end{array}$ & P value \\
\hline Miller et al & $15(8.8)$ & $<0.0001 \mathrm{~S}$ \\
\hline Eden et al (337) & $23(6.8)$ & $<0.05 \mathrm{~S}$ \\
\hline Nageotte et al & $155(5.6)$ & $<0.0001 \mathrm{~S}$ \\
\hline Present study (100) & $13(52 \%)$ & $<0.001 \mathrm{~S}$ \\
\hline
\end{tabular}


Table 3: Comparison of 5 minute APGAR score of $<9$ with other study groups.

\begin{tabular}{|lll|}
\hline Studies & $\begin{array}{l}\text { No. of patients } \\
(\%)\end{array}$ & $\begin{array}{l}\text { P value } \\
\text { Nageotte et al }\end{array}$ \\
\hline $13(0.8 \%)$ & $\begin{array}{l}\text { Not } \\
\text { significant }\end{array}$ \\
\hline Eden et al (337) & $5(1.5 \%)$ & $\begin{array}{l}<0.001, \\
\text { Significant }\end{array}$ \\
\hline Present study (100) & $5(15 \%)$ & $\begin{array}{l}<0.05 \\
\text { Significant }\end{array}$ \\
\hline
\end{tabular}

Figure 6 shows that the chances of LSCS in a patient with abnormal MBPP are statistically higher than in those with normal MBPP. In this study it is seen that the rate of caesarean section is high when NST is abnormal. Hence, patients with abnormal MBPP should be meticulously monitored and delivered where services for emergency LSCS are available round the clock.

Figure 7 shows that chances of having meconium stained liquor are more in cases of abnormal MBPP, but this corelation is not statistically significant in this study and needs further validation with a larger sample size. Also, no statistical relevance can be seen in the individual component of MBPP and presence of meconium.

The reference studies showed that there was significant meconium staining of liqour in cases of abnormal MBPP. In the present study, it showed that whenever the MBPP abnormal, we had $26 \%$ cases showing meconium which is also a significant value comparable to other studies.

Figure 8 shows that the presence of intrapartum fetal distress is significantly and statistically higher in cases with abnormal MBPP. The presence of intrapartum fetal distress is seen significantly in all cases of non reactive NST who are given trial of labour. Hence, if patients with abnormal MBPP and even more with non reactive NST are given trial of labour, they should be monitored with continuous intrapartm fetal monitoring so that fetal distress can be picked up at the earliest.

In the study by Miller et al, the incidence of fetal distress when test results were abnormal was high compared to those when MBPP was normal (36\% v/s $13.2 \%$ $\mathrm{p}<0.0001)$. Similar results were seen in the study by Eden et al, who has $15.8 \%$ fetal distress rate when test results were abnormal, compared to $4.1 \%$ when the results were normal. ${ }^{13}$ In our study, the incidence of caesarean section for fetal distress was also very high (52\%) and statistically significant. Figure 9 shows that the chances of baby having APGAR $<9$ are more when the MBPP is abnormal, but this is not statistically significant in this study. Also the chance of a baby having APGAR $<9$ has no statistical significance depending on the individual component of MBPP. This may be because, most of the patients with abnormal MBPP went in for a LSCS without trial of labour and hence, the fetus had a favourable outcome.
$15 \%$ patients with an abnormal MBPP had babies with APGAR $<9$ in this study. Whereas in earlier studies, Nageotte et al had $0.8 \%$ while Eden et al had $1.5 \%$ patients with abnormal MBPP having APGAR <9.3,13 Present studies significant value is comparable with study of Eden et al. ${ }^{13}$ Thus, the chances of the neonate needing resuscitation after birth are more when the modified BPP is abnormal and this difference is statistically significant (Figure 10). The chance of the baby needing neonatal resuscitation more with inadequate AFI, and this difference is statistically significant in this study which is comparable to Panda S et al (Figure 11). ${ }^{16}$ Also, as AFI is a marker of long term fetal condition, oligohydramnios is usually associated with need for neonatal resuscitation. Hence, an obstetrician should consider delivering a fetus with abnormal MBPP and more so with inadequate AFI in a centre with efficient facility for neonatal resuscitation.

Figure 12 shows that the perinatal morbidity is statistically more in patients with abnormal MBPP. Figure 13, also shows the fetus with inadequate AFI have statistically more chances of having perinatal morbidity as AFI is a marker of long term fetal and placental condition which is comparable to Kahkhaie $\mathrm{K}$ et al. ${ }^{17}$ Hence, all fetus with with abnormal MBPP and more so with oligohydramnios have to be delivered with centres with good perinatal care facilities, well equipped NICU and efficient neonatologists.

\section{CONCLUSION}

Modified biophysical profile (MBPP) is easier, less time consuming, cost effective and patient compliant test and hence, can be used as a primary antepartum fetal surveillance test to predict perinatal outcome and provide timely intervention in high risk pregnancies. When the Modified biophysical profile is normal, it gives reassurance that the fetal status is good with good perinatal outcome. At the same time, when MBPP is abnormal, it indicates that the fetus may be compromised.

MBPP can help the obstetrician for appropriately timed induction so as to prevent iatrogenic prematurity and also safegaurd neonatal outcome. MBPP can be used as an indicator to transfer the patient to an institute with intensive fetal monitoring availability, facility for emergency LSCS, well equipped NICU and skilled neonatologist availability.

\section{Limitations of this study}

- The number of patients included in this study was 100. To formulate a definitive protocol, further multicentric studies with larger samples should be conducted.

- The NST component of MBPP has a high false positive rate.

- $\quad$ MBPP cannot reduce the perinatal morbidity due to low birth weight. 
- The perinatal morbidity due to intrapartum fetal distress due to hyperstimulation of the uterus cannot be reduced by a pre-induction MBPP.

- Comparison between Manning's Score and MBPP is not done.

- The babies with perinatal morbidity have been followed up only till their hospital stay. Long term follow up is required to ascertain their development.

\section{ACKNOWLEDGMENTS}

Author would like to acknowledge Dr. Ranjitsinha Mane, Dr. Swaroop Borade, Dr. Akansha Barkase, for his constant encouragement and support.

\section{Funding: No funding sources}

Conflict of interest: None declared

Ethical approval: Not required

\section{REFERENCES}

1. Sankar MJ, Neogi SB, Sharma J, Chauhan M, Srivastava R, Prabhakar PK, et al. State of newborn health in India. Indian J Perinatol. 2016;36:S3-8.

2. Manning FA, Platt LD, Sipos L. Antepartum fetal evaluation: Development of a fetal biophysical profile. American J Obstet Gynecol. 1980;136(6):787-95.

3. Nageotte MP, Towers CV, Asrat T, Freeman RK. Perinatal outcome with the modified biophysical profile. Am J Obstet Gynecol. 1994;170(6):1672-6.

4. Miller DA, Rabello YA, Paul RH.The modified biophysical profile: Antepartum testing in the 1990. Am J Obstet Gynecol, 1996;174(3):812-7.

5. Jeng CJ, Jou TJ, Wang KG, Yang YC, Lee YN, Lan CC. Amniotic fluid index measurement with the four-quadrant technique during pregnancy. J Reprod Med. 1990;35(7):674-7.

6. Phelan JP, Ahn MO, Smith CV, Rutherford SE, Anderson E. Amniotic fluid index measurements during pregnancy. J Reprod Med. 1987;32:601-4.
7. Intrapartum care: NICE guideline CG190 (February 2017) National Institute for Health and Care Excellence, 2017.

8. Edmunds SF, Silver RM. Stillbirth reduction efforts and impact on early births. Clin Perinatol. 2013;40(4):611-28.

9. Donald I. Assessment of fetal wellbeing. In: Renu Misra: practical obstetric problems.6th ed, New Delhi: BI Publications Pvt Ltd; 2007: 465-485.

10. Matsura M, Murate Y, Hirano T, Sude K. The effects of developing ANS on FHR variabilities determined by the power spectral analysis. Am J Obset Gynaecol. 1996;174:380.

11. Arias F, Daftary, Bhide, Practical guide to high risk pregnancy and delivery. 3rd ed. New Delhi: Elsevier, 2008;17-22.

12. Raouf S, Sheikhan F, Hassanpour S, Bani S, Torabi $\mathrm{R}$, Shamsalizadeh N. Diagnostic value of non stress test in latent phase of labor and maternal and fetal outcomes. Glob J Health Sci. 2014;7(2):177-82.

13. Eden L, Hetzles G, Smith CV. Prenatal outcome with biophysical profile. Am J Obstet Gynaecol. 1994;170:1620.

14. Patil SK, Ghregrat RH, Khadilkar SS. Correlation of NST and AFV in antenatal fetal monitoring. J Obstet Gynecol India. 1998; 32(106):177-81.

15. Miller M, Paul RH, Rabello YA. The biophysical profile - ante partum testing in the 1990's. Am J Obstet Gynaecol. 1996;174(3):812-7.

16. Panda S, Jayalakshmi M, Shashi Kumari G, Mahalakshmi G, Srujan Y, Anusha V. Oligoamnios and Perinatal Outcome. J Obstet Gynaecol India. 2017;67(2):104-8.

17. Rezaie Kahkhaie K, Keikha F, Rezaie Keikhaie K, Abdollahimohammad A, Salehin S. Perinatal outcome after diagnosis of oligohydramnious at term. Iran Red Crescent Med J. 2014;16(5):e11772.

Cite this article as: Borade JS, Sharma SP. The role of modified biophysical profile in predicting perinatal outcome in high risk pregnancies. Int J Reprod Contracept Obstet Gynecol 2018;7:2287-94. 